\title{
Guanylate-binding protein-1 is expressed at tight junctions of intestinal epithelial cells in response to interferon- $\gamma$ and regulates barrier function through effects on apoptosis
}

\author{
M Schnoor $^{1,2}$, A Betanzos ${ }^{1,2}$, DA Weber ${ }^{1}$ and CA Parkos ${ }^{1}$
}

Guanylate-binding protein-1 (GBP-1) is an interferon inducible large GTPase involved in endothelial cell proliferation and invasion. In this report, expression and function of GBP-1 were investigated in vitro in intestinal epithelia after exposure to interferon- $\gamma$ and in human colonic mucosa from individuals with inflammatory bowel disease (IBD). Interestingly, in contrast to other epithelia, GBP-1 distributed to the plasma membrane in intestinal epithelial cells where it colocalized with the tight junction protein coxsackie- and adenovirus receptor. In addition, expression of GBP-1 was upregulated in colonic epithelia of individuals with IBD. Downregulation of GBP-1 by siRNA resulted in enhanced permeability that correlated with increased apoptosis. Indeed, inhibition of caspase activity prevented the inhibition of barrier formation induced by the loss of GBP-1. These data suggest that GBP-1 is a novel marker of intestinal mucosal inflammation that may protect against epithelial apoptosis induced by inflammatory cytokines and subsequent loss of barrier function.

\section{INTRODUCTION}

Guanylate-binding protein (GBP)-1 is one of the genes most strongly induced by interferons (IFNs). ${ }^{1}$ GBP-1 belongs to a family of GTPases which are classified into three groups: (1) the large GTPases, also known as GBPs; (2) the small GTPases; and (3) the Mx proteins. ${ }^{2}$ The human large GTPase family consists of seven members encoded by a gene cluster located on chromosome $1 .^{3}$ The crystal structure of GBP-1 has revealed two different domains: a compact globular domain at the $\mathrm{N}$ terminus containing the GTPase function and an $\alpha$-helical domain at the C terminus. ${ }^{4}$

GBP-1 is highly expressed in endothelial cells activated by inflammatory cytokines in vitro and in vivo and it regulates the inhibition of proliferation and invasion of endothelial cells in response to IFN $-\gamma .^{5-7}$ GBP- 1 has been shown to exert antiviral activity against vesicular stomatitis virus and encephalomyocarditis virus. ${ }^{8}$ Downregulation of GBP-1 by means of siRNA resulted in higher levels of hepatitis $\mathrm{C}$ virus replication. ${ }^{9}$ In addition to its GTPase function and its involvement in viral infections, GBP-1 also contributes to cell survival as overexpression of GBP-1 caused inhibition of apoptosis in human umbilical vein endothelial cells after growth factor and serum depletion. ${ }^{10}$ GBP-1 can be isoprenylated at a CaaX-motif at the very C-terminal end. ${ }^{4,11}$ Recently, it has been demonstrated in HeLa cells that overexpressed GBP-1 translocates to the Golgi membrane after IFN- $\gamma$ stimulation. This translocation required nucleotide binding, isoprenylation, and IFN induction of the cells. ${ }^{12}$ However, despite the isoprenylation motif, GBP-1 has never been shown to be located at the plasma membrane.

Very little is known about GBP-1 expression and function in human epithelial cells. ${ }^{6,13}$ In epithelial cells, several groups have demonstrated that IFN- $\gamma$ treatment can lead to apoptosis and compromised barrier function through different effects that may include sensitization to other inflammatory cytokines and/or FAS ligand. ${ }^{14-17}$ In intestinal mucosal tissue, chronic inflammatory conditions may manifest as inflammatory bowel disease (IBD), a chronic, relapsing disorder of the gastrointestinal tract. ${ }^{18,19}$ There are two major forms of IBD: Crohn's disease (CD) and ulcerative colitis (UC), both of which lead to long-term, sometimes irreversible damage of the gastroin-

1Epithelial Pathobiology Unit, Department for Pathology and Laboratory Medicine, Emory University, Atlanta, Georgia, USA. ${ }^{2}$ These authors contributed equally to this work. Correspondence: M Schnoor (mschnoo@emory.edu) or C Parkos (cparkos@emory.edu)

Received 2 July 2008; accepted 14 August 2008; published online 17 September 2008. doi: 10.1038/mi.2008.62 
testinal structure and function. The pathogenesis of IBD is still poorly understood. However, it is known that multiple factors are implicated, such as genetic variations, altered gut flora, and a defective immuneregulation. ${ }^{18-21}$ Of note, IBD is characterized by mucosal tissue damage, apoptosis, and increased levels of proinflammatory cytokines such as the GBP-1-inducing interferons. Proinflammatory cytokines cause altered expression profiles in leukocytes and epithelial cells that affect the epithelial barrier. ${ }^{18}$ Genes regulated by inflammatory cytokines are therefore of great importance as targets for the development of new ideas for IBD treatment.

We hypothesized that GBP-1 may be induced under inflammatory conditions in intestinal epithelial cells and that it may be involved in regulating barrier function. We found increased GBP-1 levels in IFN- $\gamma$-treated human intestinal epithelial cells, in colonic mucosa of individuals with IBD, and we observed a localization of GBP-1 at tight junctions (TJs) exclusively in intestinal epithelia. In addition, GBP-1 downregulation by siRNA inhibited epithelial barrier formation due to a higher susceptibility to apoptosis under inflammatory conditions. The implications of these findings with respect to mucosal protective mechanisms are discussed.

\section{RESULTS}

\section{Human epithelial cell lines express GBP-1 after IFN- $\gamma$} treatment

Different human epithelial cell lines that were treated with $100 \mathrm{U} / \mathrm{ml}$ IFN- $\gamma$ or untreated were screened for the expression of GBP-1 mRNA using intron-spanning primers to avoid amplification of genomic DNA. As a positive control, we used untreated and IFN- $\gamma$-treated 14-day-old human macrophages. By semiquantitative RT-PCR analyses, all cells tested showed an increase in GBP-1 mRNA level after IFN- $\gamma$ treatment (Figure 1a). However, the strongest upregulation of message was observed in the cervical epithelial cell lines Caski and HeLa, and in the retinal epithelial cell line ARPE all of which expressed very low levels of GBP-1 mRNA in the resting state. By contrast, the intestinal epithelial cell lines T84, HT29, and SK-CO15 as well as human macrophages all exhibited detectable levels of GBP-1 mRNA in the untreated state and had variable increases in mRNA levels after IFN- $\gamma$ treatment. The increase in message was most pronounced in T84 cells.

Next, GBP-1 protein expression was investigated by western blot (Figure 1b). Protein levels of GBP-1 were very low to absent in all untreated cells tested. After IFN- $\gamma$-treatment, there was a strong increase of GBP-1 protein in intestinal as well as in nonintestinal epithelial cells. However, we observed differences in mRNA and protein amounts especially in the resting state. Such discrepancies in the relative amounts of proteins and their mRNAs are difficult to explain, but certainly not unusual. Others have shown that only approximately $50 \%$ of observed changes at the protein level actually correlate with corresponding mRNA levels. The authors discuss such cases and give possible explanations. ${ }^{22}$

In subsequent functional studies, SK-CO15 cells were $\mathrm{u}$ sed as this transformed cell line forms high resistance, polarized monolayers, and is readily transfectable. ${ }^{23}$ Interestingly, SK-CO15 cells had the lowest amount of GBP-1. However, coincubation with IFN- $\gamma$ and tumor necrosis factor (TNF)- $\alpha$ enhanced GBP-1 expression (Figure 1c), presumably due to TNF- $\alpha$-induced enhancement of IFN- $\gamma$ receptor expression. ${ }^{24}$ However, TNF- $\alpha$ alone did not induce GBP-1 expression. a

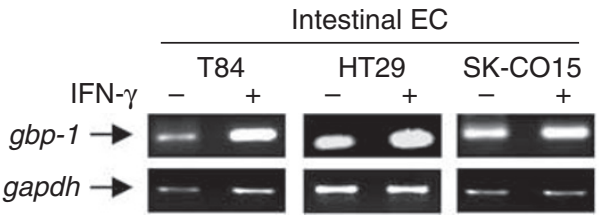

b

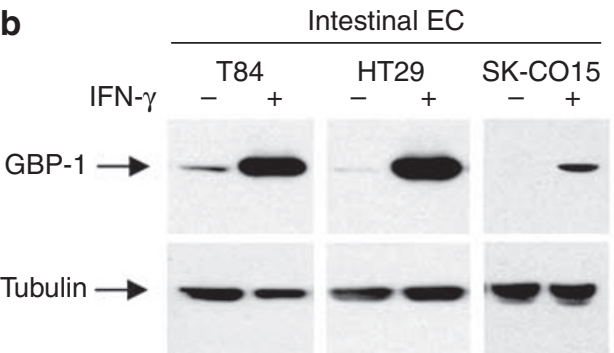

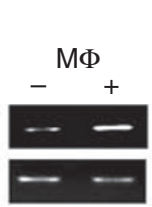
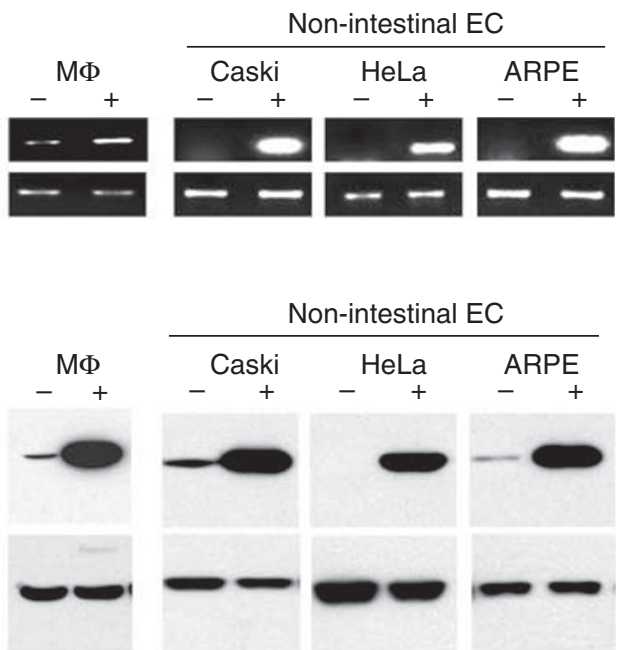

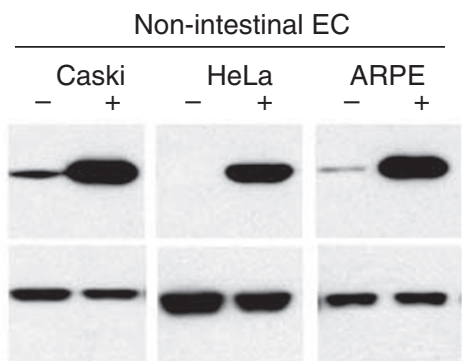

C

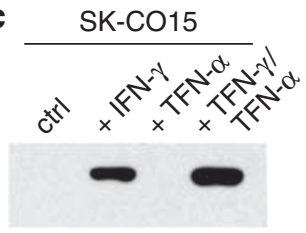

Figure 1 Human epithelial cell lines express guanylate-binding protein-1 (GBP-1) mRNA and protein after interferon (IFN)- $\gamma$ treatment. (a) Reverse transcriptase (RT)-PCR analysis of GBP-1 expression. Intestinal as well as nonintestinal epithelial cell (EC) lines show strong expression of GBP-1 mRNA after IFN- $\gamma$ treatment for $24 \mathrm{~h}$. Macrophage (MФ) CDNA was used as a positive control, glyceraldehyde 3-phosphate dehydrogenase as loading control. (b) Western blot analysis of GBP-1 expression. All EC lines tested show strong production of GBP-1 protein after IFN- $\gamma$ treatment. M $\Phi$ cell lysates served as positive control and tubulin blots as loading control. (c) Tumor necrosis factor (TNF)- $\alpha$ increases the susceptibility of SK-CO15 cells to IFN- $\gamma$. However, TNF- $\alpha$ alone does not induce GBP-1 expression. Displayed are representative images of three independent PCR and western blot experiments. 
GBP-1 is localized at TJs in intestinal epithelial cells after IFN- $\gamma$ treatment

The intracellular localization of GBP-1 was investigated using immunofluorescence (IF) staining and confocal laser microscopy. Human macrophages were used as positive controls and showed strong staining in the cytosol after IFN- $\gamma$ treatment (Figure 2a). Untreated cervical epithelial cell lines HeLa and Caski showed no GBP-1 staining, whereas the untreated retinal epithelial cell line ARPE showed weak cytosolic staining (Figure 2a). After IFN- $\gamma$ treatment, Caski and ARPE cells showed very strong cytosolic staining, whereas HeLa cells showed a lower amount of GBP-1 compared with the other cell lines (Figure 2a). The TJ molecule coxsackie- and adenovirus receptor (CAR) was stained to visualize the intercellular junctions. CAR belongs to the CTX family of transmembrane proteins and was originally identified as a virus receptor before its location at the TJ and its function as adhesion molecule were discovered. ${ }^{25}$ At the TJ, CAR has been shown to be involved in regulating epithelial barrier function and in homotypic intercellular interactions. No colocalization of GBP-1 and CAR could be detected in the above cell lines.

Intriguingly, the expression pattern of GBP-1 was strikingly different in intestinal epithelial cell lines. All three intestinal cell lines T84, SK-CO 15, and HT29 showed weak staining for GBP-1 in the resting state (Figure 2b). However, after IFN- $\gamma$ treatment or IFN- $\gamma+$ TNF- $\alpha$ for SK-CO15 cells, there was strong lateral membrane staining that colocalized with CAR (Figure 2b). In addition, the CAR expression did not seem to change in these cell lines after $24 \mathrm{~h}$ exposure to IFN- $\gamma$, as it was previously reported for endothelial cells. ${ }^{26}$ This finding was corroborated in $x z$ images that also showed colocalization at TJs (Figure 2b). Such intercellular staining was unexpected, given that GBP-1 has not been previously reported to localize to the intercellular junctions.

To independently confirm the presence of GBP-1 at the plasma membrane, cell fractionation experiments were performed using T84 cells that were treated with IFN- $\gamma$ (Figure 3a). The purity of subcellular fractions (membrane, nuclei, and cytosol) was assayed by western blot for specific marker proteins (Figure 3b). Aliquots of the total cell lysates before fractionation were probed for GBP-1 and demonstrated upregulation of GBP-1 protein levels after IFN- $\gamma$ treatment. Nuclear fractions contained no GBP-1 protein. Western blots of cytosol fractions showed weak bands for GBP-1 in untreated cells and strong upregulation of protein expression after IFN- $\gamma$ treatment. Of note, western blots of membrane fractions of IFN- $\gamma$-treated cells also showed strong GBP-1 expression (Figure 3a). These findings suggest that GBP1 translocates from the cytosol to intercellular junctions under inflammatory conditions in intestinal epithelial cells.

\section{GBP-1 localizes to TJs in human colonic mucosa from individuals with IBD}

As proinflammatory cytokines are elevated in mucosal tissues from individuals with IBD, we investigated whether GBP-1 is also upregulated in these tissues. IF staining revealed minimal GBP-1 expression in normal control tissue without signs of inflamma- tion, whereas a strong upregulation of GBP-1 was observed in colonic epithelia of individuals with active UC (Figure 4). GBP1 was also observed to be upregulated in mucosa of individuals with active CD but to a slightly lesser degree than in UC. We performed fluorescence pixel intensity analyses for each condition using LSM-FCS software (Zeiss, Jena, Germany) and obtained the following results (arbitrary units of pixel intensity): control tissue: $13.72 \pm 4.94$; UC: $72.63 \pm 13.37$; and CD: $50.36 \pm 10.5$. Compared with control GBP-1 staining, the increases in both UC and CD are highly significant with $P$-values of $\leqslant 0.001$.

In a fashion similar to that observed in vitro, GBP-1 colocalized with CAR at the TJ in mucosal tissue samples of individuals with active CD and UC. By contrast, the small amount of GBP-1 detectable in control tissue was present in the cytosol only.

\section{Downregulation of GBP-1 expression by siRNA inhibits formation of the epithelial barrier}

Given the colocalization of GBP-1 with intestinal epithelial TJs, we speculated that GBP-1 may be involved in regulating epithelial barrier function. To test this hypothesis, we employed a gene-silencing approach after $24 \mathrm{~h}$ of IFN- $\gamma$ treatment with specific downregulation of GBP-1 by transient transfection with siRNA. The specificity of GBP-1 downregulation was demonstrated by western blot and IF staining (Figure 5a). Cells in which cyclophilin $\mathrm{B}(\mathrm{CyB})$, a gene not related to barrier function, was silenced served as controls. Transfection of SK-CO15 cells with GBP-1 siRNA resulted in a complete removal of GBP-1 from intercellular junctions (Figure 5a). The development of transepithelial electrical resistance (TER) was monitored every $24 \mathrm{~h}$ in mock-transfected cells and in cells transfected with CyB or GBP-1 siRNA. Development of TER was significantly impaired in cells transfected with GBP-1 siRNA compared with mock-transfected cells and cells transfected with CyB siRNA (Figure 5b). TER development of SK-CO15 cells after siRNAtransfection but without IFN- $\gamma$ treatment has been previously reported by our group and showed a generally higher TER over time than the IFN- $\gamma$ treated cells in the present study. ${ }^{23}$

As a complementary assessment of permeability, paracellular flux of $4 \mathrm{kDa}$ fluorescein isothiocyanate-labeled dextran was measured in the same experimental setup. After transfection with GBP-1 siRNA and determination of effects on TER, dextran was added and flux measured $24 \mathrm{~h}$ later. As expected, greater than a twofold increase in paracellular flux was detected in cells transfected with GBP-1 siRNA compared with controls (Figure 5c). These findings support the notion that GBP-1 loss is detrimental to barrier function under inflammatory conditions.

\section{Downregulation of GBP-1 expression by siRNA enhances apoptosis}

In order to determine the mechanism of altered barrier function after GBP-1 knockdown, SK-CO15 cells were screened for signs of apoptosis after IFN- $\gamma$ and GBP-1 or CyB siRNA treatment. Transfected cells were harvested when differences in TER were observed and probed for different apoptosis markers by western blot (Figure 6a). Tubulin blots served as loading 
a

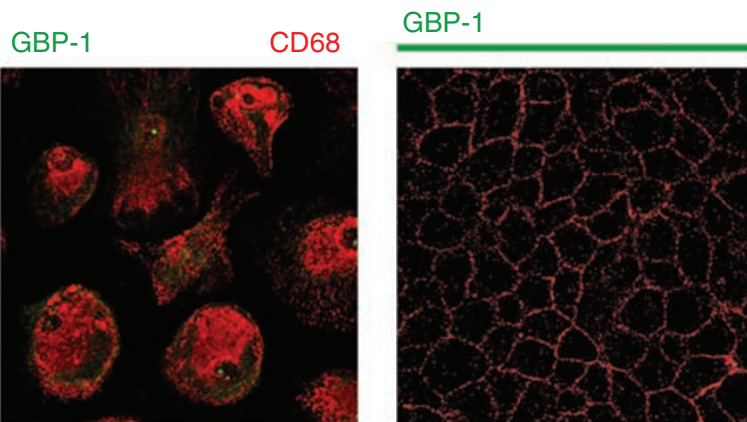

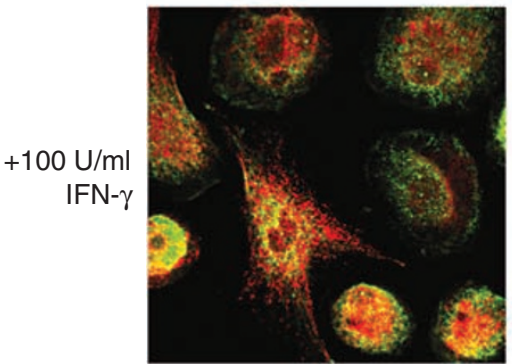

МФ

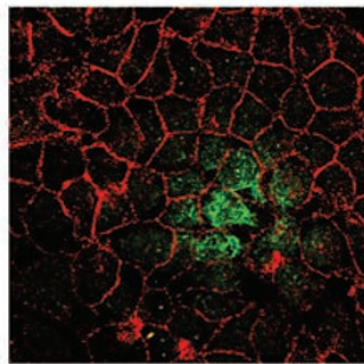

HeLa
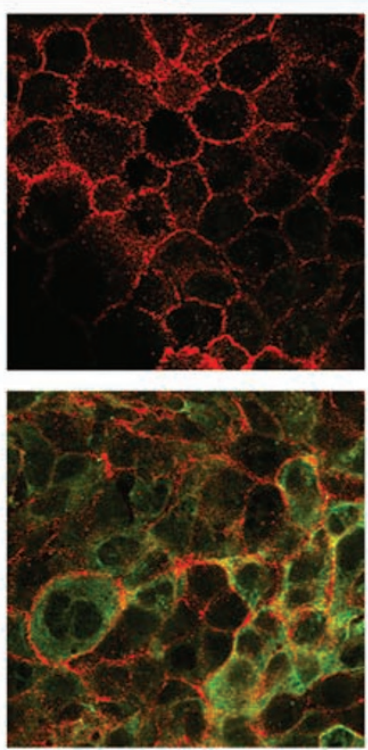

Caski
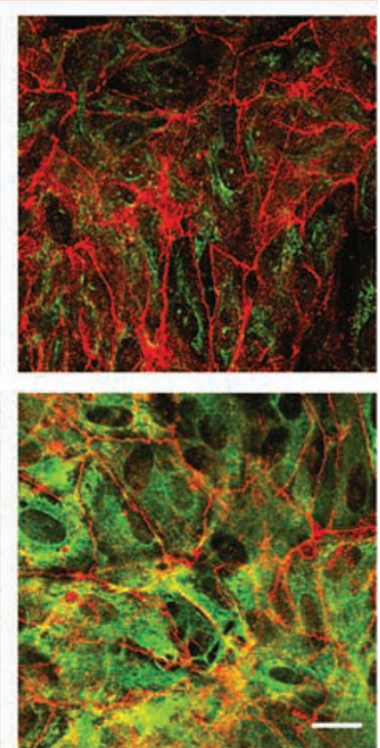

ARPE-19

b

Coxsackie- and adenovirus receptor (CAR)
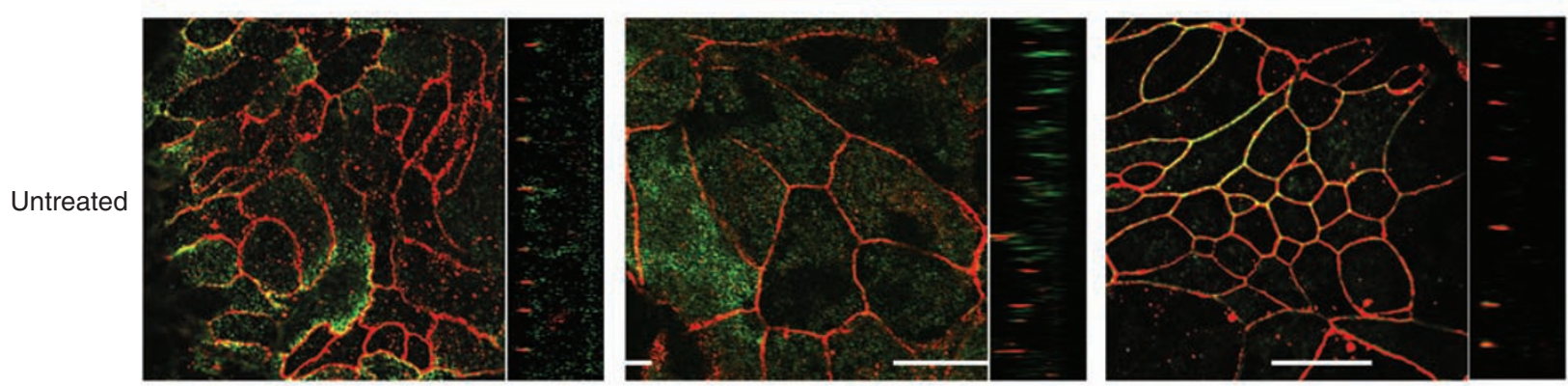

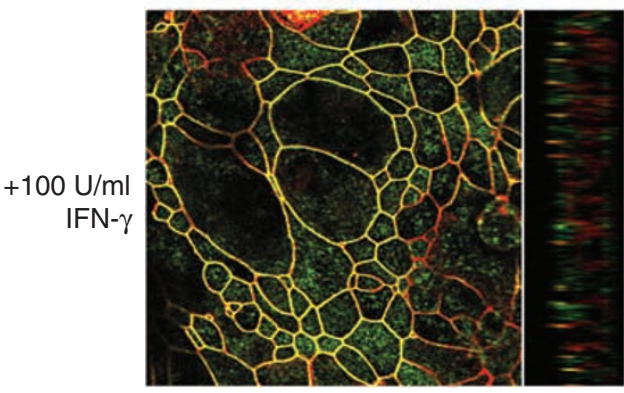

T84

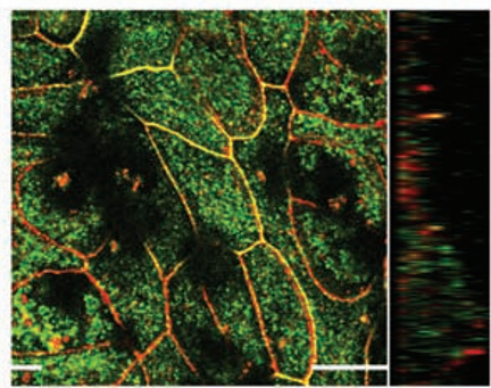

SK-C015

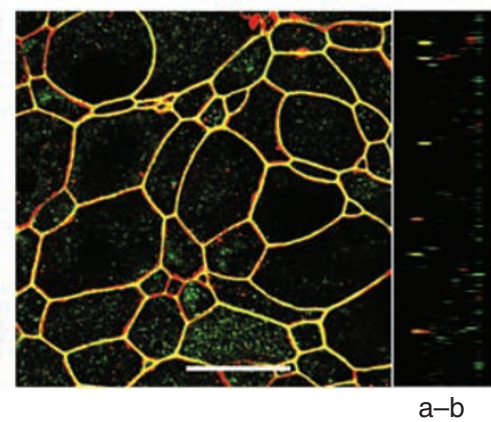

HT29

Figure 2 Guanylate-binding protein-1 (GBP-1) is localized at tight junctions (TJ) in intestinal epithelial cells after interferon (IFN)- $\gamma$ treatment. (a) Immunofluorescence (IF) staining of M $\Phi$ and nonintestinal epithelial cell lines show a strong increase of GBP-1 expression after IFN- $\gamma$ treatment. However, GBP-1 is only detected in the cytosol. (b) In intestinal epithelial cells, GBP-1 is not only induced after IFN- $\gamma$ treatment but a great portion of the produced GBP-1 can be detected at intercellular junctions colocalizing with the TJ molecule coxsackie- and adenovirus receptor (CAR). Images of the $x z$ axes corroborate TJ localization of GBP-1 in intestinal epithelial cell lines (a-b: apical-basal). Bar $=20 \mu \mathrm{m}$.

controls. Apoptosis markers investigated were poly-(ADPribose)-polymerase (PARP), caspase-3, and caspase-9 as cleavage of these molecules is an early sign of apoptosis. PARP is a major target for cleavage by active caspase-3, which in turn is cleaved by caspase- $9 .{ }^{27}$ The protein band for the large PARPfragment $(\Delta$-PARP) was enhanced in cells transfected with GBP-1 siRNA to a greater extent than in those transfected with
CyB siRNA (Figure 6a). In addition, the proenzymes caspases 9 and 3 were cleaved, thus representing more activation in cells treated with GBP-1 siRNA (Figure 6a). Taken together, these findings indicate increased susceptibility to apoptosis after GBP1 downregulation. To corroborate these findings, we performed terminal transferase dUTP nick-end labeling (TUNEL) staining (Figure 6b). SK-CO15 cells transfected with GBP-1 siRNA 
displayed stronger TUNEL labeling than those transfected with CyB siRNA. Quantification of labeled cells demonstrated more than a twofold increase in TUNEL labeling of cells trans-

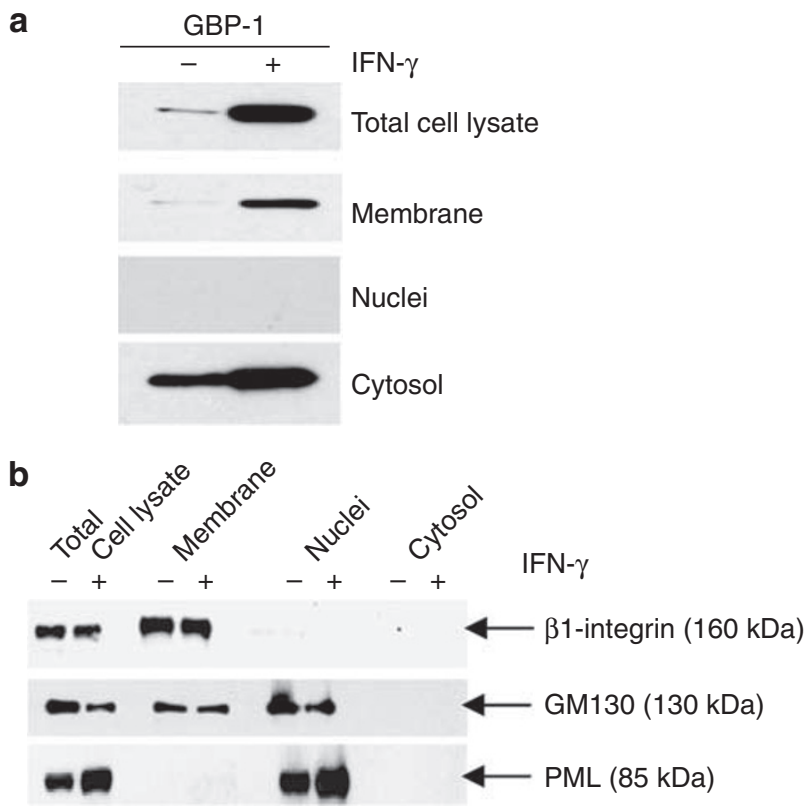

Figure 3 Guanylate-binding protein-1 (GBP-1) cosediments with membrane fractions after treatment with interferon (IFN)- $\gamma$. (a) Cellular fractionation of untreated or IFN- $\gamma$-treated T84 cells and western blot analysis revealed that GBP-1 is present in the membrane fraction of IFN- $\gamma$-treated cells. (b) Western blots confirmed the purity of the fractions. $\beta 1$-Integrin was only detected in the membrane fraction and promyelocytic leukemia protein (PML) only in the nuclear fraction. The absence of $\beta 1$-integrin and the Golgi marker 130 (GM130) from the cytosolic fraction confirmed that the cytosolic fraction contained no membrane residues. fected with GBP-1 siRNA compared with controls (Figure 6b). Addition of the caspase inhibitor Z-VAD to cells transfected with GBP-1 siRNAs protected these cells from apoptosis. In addition, IF staining for active caspase- 3 was assessed and corroborated the TUNEL results (data not shown).

To determine whether caspase-mediated apoptosis is indeed a mechanism for altered barrier function, we performed TER measurements in cells transfected with GBP-1 or CyB siRNA that were additionally treated with the caspase inhibitor Z-VAD. Caspase inhibition reversed the impaired barrier function in cells transfected with GBP-1 siRNA. By contrast, control cells were unaffected by the addition of Z-VAD (Figure 6c).

\section{DISCUSSION}

The importance of GBP-1 in response to inflammatory stimuli has previously been studied by others in different cell types where expression of GBP- 1 was induced by proinflammatory cytokines. ${ }^{1,6,13,28-30}$ In this study, we demonstrated expression of GBP-1 in cultured intestinal epithelial cells after exposure to IFN- $\gamma$ and in colonic epithelia of individuals with IBD. Strikingly, an intestinal epithelial cell-specific association of GBP-1 with the lateral cell membrane and colocalization with the TJ marker CAR was observed. Importantly, loss of GBP-1 expression negatively affected the development of epithelial barrier function, presumably due to increased apoptosis induced by proinflammatory cytokines.

GBP-1 was detected at intestinal epithelial TJs and long-term treatment with IFN- $\gamma$ has previously been shown to mediate intestinal epithelial barrier disruption in vitro and in vivo and also apoptosis possibly due to sensitization to other proinflammatory cytokines such as TNF- $\alpha .{ }^{15,17,24}$ Thus, we speculated that GBP-1 expression under inflammatory conditions may initially
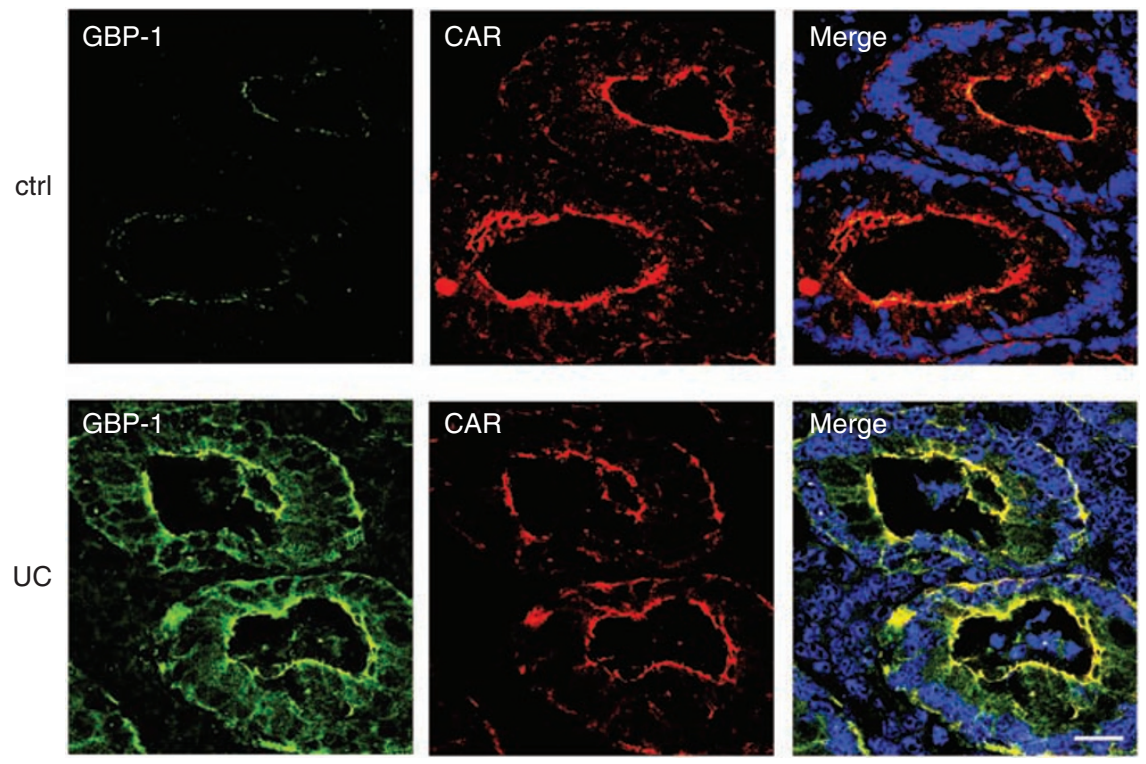

Figure 4 Guanylate-binding protein-1 (GBP-1) is upregulated in colonic mucosa of individuals with ulcerative colitis (UC). Human colon tissue sections $(n=3)$ of individuals with active UC or Crohn's disease and control tissue (ctrl) without inflammation were labeled with monoclonal GBP-1 (green) and coxsackie- and adenovirus receptor (CAR) (red) antibodies. Very little GBP-1 is expressed in the control tissue, whereas UC tissue shows a strong staining of GBP-1 in crypt epithelium in colocalization with CAR. The displayed image of the UC tissue is representative for the results obtained with all inflammatory bowel disease (IBD) tissue samples. Nuclei are shown in blue. Bar $=20 \mu \mathrm{m}$. 

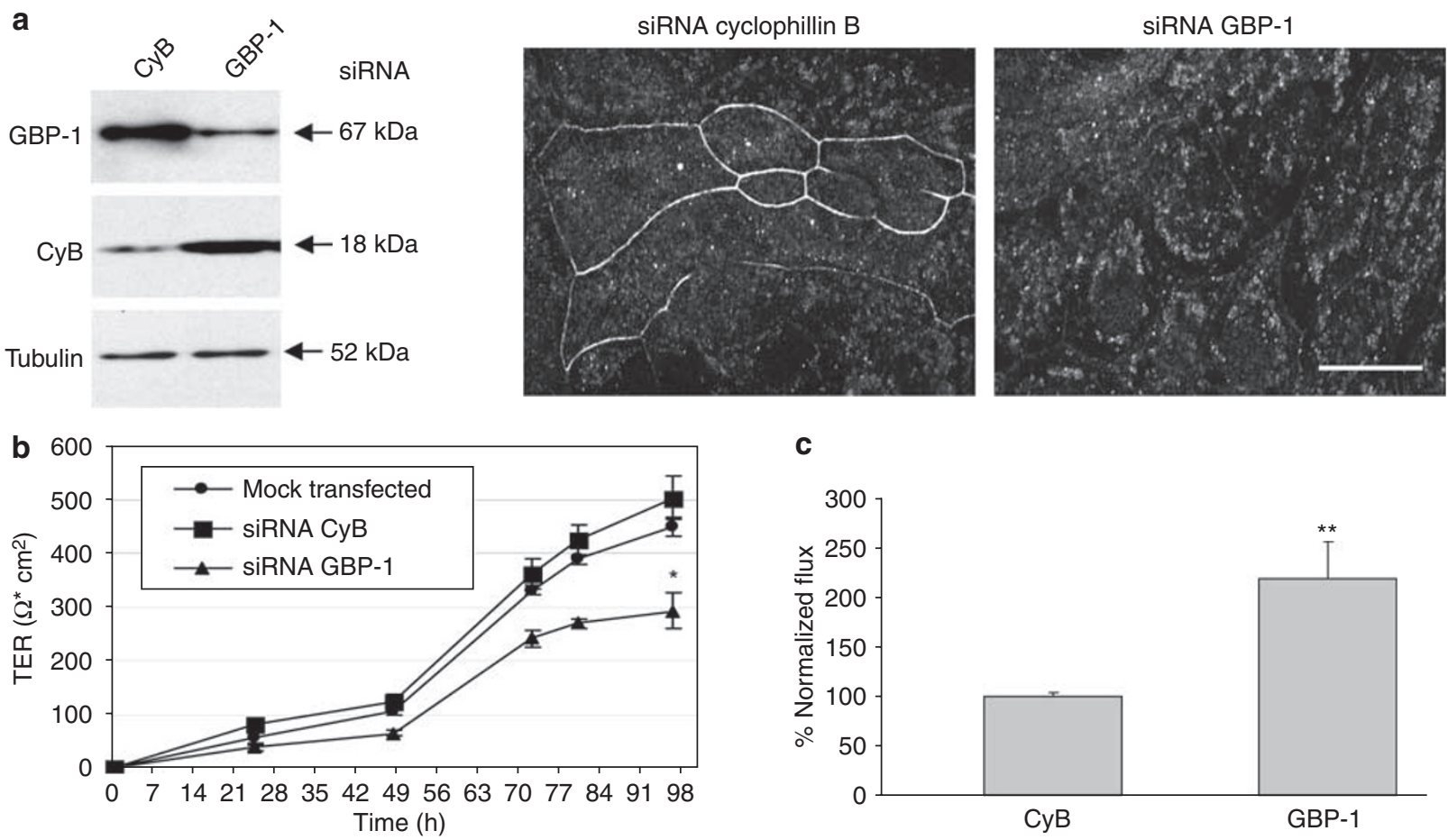

Figure 5 Guanylate-binding protein-1 (GBP-1) downregulation inhibits epithelial barrier formation. (a) Downregulation of GBP-1 by siRNA is efficient and specific in SK-CO15 cells treated with interferon (IFN)- $\gamma$ and tumor necrosis factor (TNF)- $\alpha$. Western blot analyses show silencing of GBP-1 in cells transfected with GBP-1-specific siRNA, whereas cells transfected with siRNA specific for cyclophilin B (CyB) show no unspecific silencing. Tubulin is shown as a loading control. The immunofluorescent (IF) image shows specific removal of GBP-1 from the lateral cell membrane. Bar $=20 \mu m$. (b) SK-CO15 cells were transfected with siRNA specific for CyB or GBP-1 after $24 \mathrm{~h}$ exposure to IFN- $\gamma$ and TNF- $\alpha$ and transepithelial electrical resistance (TER) was measured every $24 \mathrm{~h}$ in five independent experiments $(n=5)$. Cells transfected with GBP-1 siRNA showed significantly lower TER compared with cells transfected with CyB siRNA $96 \mathrm{~h}$ after transfection $\left({ }^{*} P<0.05\right)$. (c) SK-CO15 cells were transfected with siRNA specific for CyB or GBP-1. After differences in TER were measured fluorescein isothiocyanate (FITC)-labeled $4 \mathrm{kDa}$ dextran was added to the apical side and $24 \mathrm{~h}$ later the flux was fluorimetrically assessed in four independent experiments $(n=4)$. Paracellular flux was increased more than twofold in cells transfected with GBP-1 siRNA compared with cells transfected with CyB siRNA $\left({ }^{\star \star} P<0.01\right)$.

be an epithelial response to maintain barrier function. Indeed, at least in vitro, GBP-1 protects epithelial barrier function by preventing apoptosis. However, the presence of GBP-1 at intercellular junctions in colonic mucosal tissue of individuals with IBD suggests that there are long-term compensatory mechanisms for GBP-1 expression in chronically inflamed states with altered barrier function. Whether GBP-1 has also a protective function in early stages of IBD remains to be investigated. A protective effect of GBP-1 may either be short-lived in IBD or, more likely, the negative, long-term effects of IFN- $\gamma$ in IBD including the sensitization to TNF- $\alpha$ may outweigh the protective effect of GBP-1. Different functions of GBP-1 after either short- or longterm exposure to IFN- $\alpha$ have also been observed by others in endothelial cells. ${ }^{10}$ Effects of GBP-1 expression after short-term exposure to IFN- $\alpha$ resulted in decreased apoptosis. This finding is consistent with our data that GBP-1 loss after short-term treatment with IFN- $\gamma$ causes decreased barrier function due to increased apoptosis. According to these findings, both IFN- $\gamma$ and IFN- $\alpha$ have comparable beneficial short-term effects. By contrast, long-term effects of IFN- $\alpha$ treatment resulted in a senescent endothelial cell phenotype accompanied by reduced cell proliferation, ${ }^{10}$ which has also been observed by others. ${ }^{5}$ Although GBP-1 expression after short-term treatment with interferons seems beneficial to endothelial and also epithelial cell function, long-term exposure to interferons seems to be generally accompanied by decreased cell function and expression of GBP-1 is no longer protective.

Given the unique localization of GBP-1 at intercellular junctions of intestinal epithelial cells, it is likely that GBP-1 function may be different from that in other cell types in which GBP-1 expression is restricted to the cytosol. Thus, it is tempting to speculate that GBP-1 has tissue-specific functions. In particular, the intestinal epithelium is highly specialized with regard to barrier function and immune response to myriads of commensal and sometimes pathogenic microorganisms. For example, under hypoxic conditions, the intestinal epithelium has been shown to maintain barrier function in contrast to responses observed in other epithelia. ${ }^{31}$ Therefore, GBP- 1 expression at TJs might be a factor exploited by intestinal epithelial cells to protect against pathophysiologic conditions. However, additional studies investigating GBP-1 function and expression in different organs under different conditions are required.

Recently, murine GBP-1 (mGBP-1) was identified as a major target gene in the interleukin (IL)-10 - / - model of mouse colitis on different genetic backgrounds $(\mathrm{C} 3 \mathrm{H} / \mathrm{HeJ}, \mathrm{C} 57 \mathrm{BL} / 6 \mathrm{~J}) .^{32}$ The authors identified 20 genes as candidate genes by gene chip 
a

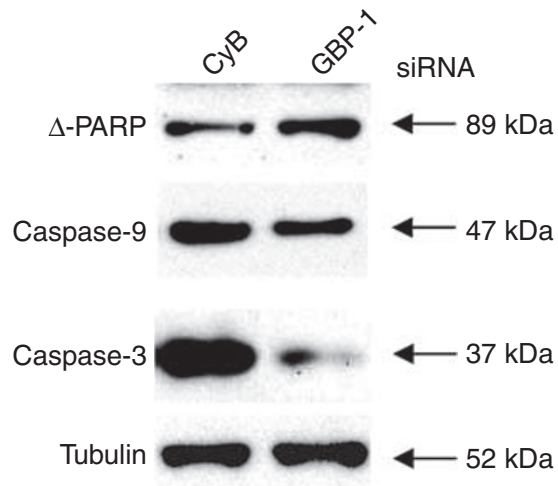

C

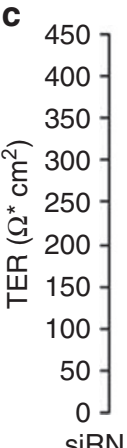

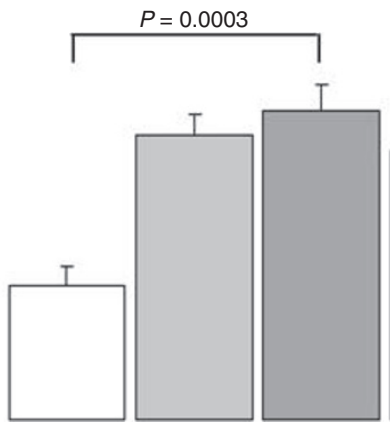

GBP-1 GBP-1
CyB b
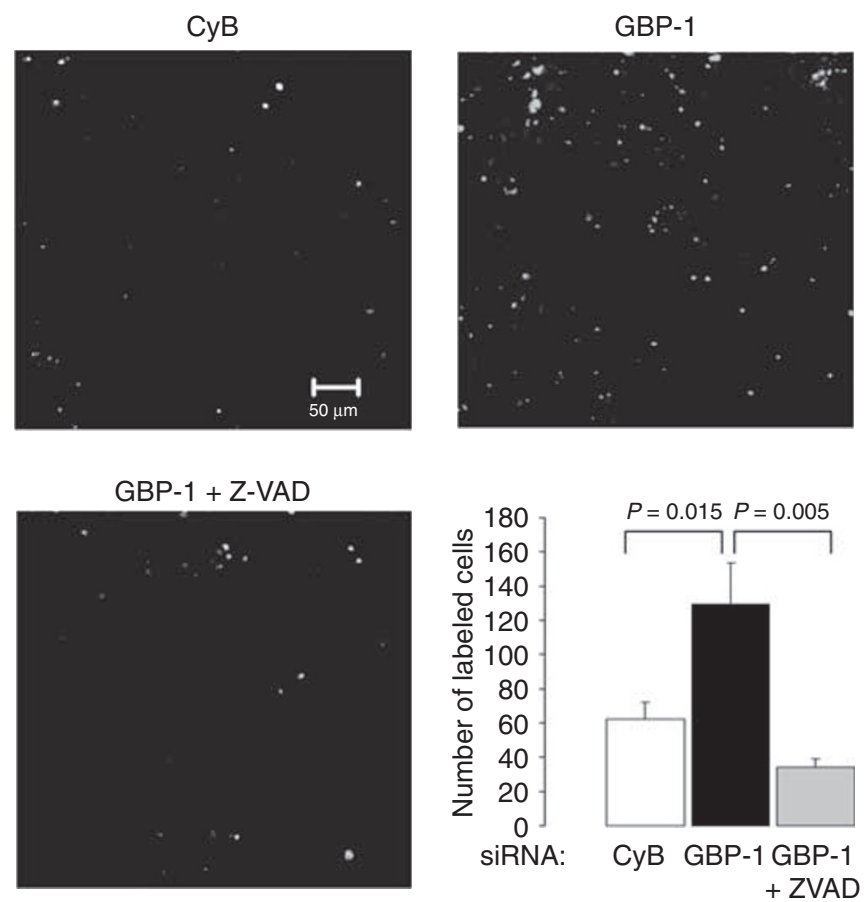

CyB

+ ZVAD

Figure 6 Guanylate-binding protein-1 (GBP-1) downregulation promotes apoptosis. (a) Apoptosis was analyzed by western blot using different markers (poly-(ADP-ribose)-polymerase (PARP), caspases 3 and 9). GBP-1 silencing increases the susceptibility of SK-CO15 cells to apoptosis as all, PARP and procaspases 3 and 9 are cleaved to a greater extent compared with cells transfected with cyclophilin B (CyB) siRNA. Tubulin was used as a loading control. (b) TUNEL staining of SK-CO15 cells transfected with either CyB- or GBP-1-specific siRNA. The images shown are representative fields of the stained filters. The graph represents quantification of data derived from five different fields. SK-CO15 cells transfected with GBP-1 siRNA show a more than twofold increase in TUNEL staining compared with controls. When treated with the caspase inhibitor Z-VAD, these cells are protected from apoptosis. (c) Cells transfected with either CyB or GBP-1 siRNA were treated with Z-VAD or left untreated. Z-VAD treatment prevents the inhibition of TER development in cells transfected with GBP-1 siRNA, whereas control cells are not affected by Z-VAD treatment.

analyses, due to their location within a colitis-susceptible quantitative trait loci interval and function in the immune response. The authors reported that the effect of the genetic background on the expression levels was important for three genes, one of which was mGBP- $1 .{ }^{32}$ In this context, three observations are important about mGBP-1: First, the mGBP-1 gene maps to the interval of the main colitogenic quantitative trait loci on mouse chromosome $3 .^{32-34}$ Second, it is highly upregulated in IFN- $\gamma$ stimulated murine cells. ${ }^{35}$ Third, GBP-1 is already known to be polymorphic between different murine inbred strains: mGBP-1 is inducible in $\mathrm{C} 3 \mathrm{H} / \mathrm{HeJ}$ (colitis-susceptible) mice whereas it is not in C57BL/6J (colitis-resistant) mice. ${ }^{36}$ In addition, mGBP1 has been shown to be induced in mice after infection with different microorganisms. ${ }^{37}$ The authors concluded that GBP1 expression in this mouse model is part of the host defense mechanism as altered gut flora and microbial infection have also been shown to be involved during the pathogenesis of IBD. ${ }^{18}$ According to these findings in mouse models of colitis and to our finding that GBP-1 is localized at colonic epithelial TJs in individuals with IBD, we hypothesize that GBP-1 may be important in regulating intestinal epithelial barrier function in IBD.

In conclusion, our findings suggest that GBP-1 expression is an initial protective event against apoptosis induced by proinflammatory cytokines in intestinal epithelia. These findings open a new direction for understanding GBP-1 function and point to a critical role of GBP- 1 in the response to intestinal mucosal inflammation. Thus, GBP-1 may be an important target gene for IBD therapies and other pathologic conditions with mucosal inflammation. The manipulation of GBP-1 expression or potential signaling mechanisms might be beneficial in early stages of acute inflammation before progression to a chronic-damaged state.

\section{MATERIALS AND METHODS \\ Cell culture}

Human intestinal (T84, HT29), human retinal (ARPE-19), and human cervical epithelial cell lines (HeLa, CaSki) were obtained from ATCC (Manassas, VA) and cultured according to the provided protocols. The human intestinal epithelial cell line SK-CO15 was a gift from Dr Enrique Rodriguez-Boulan (Weill Medical College, Cornell University, NY) and cultured as described. ${ }^{38}$ During the last $24 \mathrm{~h}$ before harvesting, all cells were incubated in the presence or absence of $100 \mathrm{U} / \mathrm{ml}$ IFN- $\gamma$ $(+10 \mathrm{ng} / \mathrm{ml}$ TNF- $\alpha$ (BD Biosciences, Franklin Lakes, NJ) for SK-CO15 cells). 
Human monocytes were isolated from whole blood of healthy human volunteers using Ficoll/dextran sedimentation as previously described. ${ }^{39}$ The procedure for leukocyte isolation was approved by Emory's Institutional Review Board. Human monocyte-derived macrophages were obtained as previously described by culturing $1.8 \times 10^{6}$ monocytes per six-well in RPMI 1640 (Sigma, St Louis, MO) containing 20\% human serum (Lonza, Walkersville, MD), $1 \%$ nonessential amino acids, $1 \%$ sodium pyruvate, and $0.1 \mathrm{mg} / \mathrm{ml}$ penicillin/streptomycin/L-glutamine (Sigma) for 14 days. ${ }^{40}$

\section{RT-PCR}

Total RNA was isolated from cell lysates using the RNeasy Mini Kit (Qiagen, Valencia, CA). cDNA was synthesized by reverse transcription using oligo $\left(\mathrm{dT}_{12-18}\right)$ primers and superscript II reverse transcriptase (Invitrogen, Carlsbad, CA). PCRs were performed using Taq-DNA polymerase (Roche, Indianapolis, IN). To avoid PCR amplification of genomic DNA, forward and reverse primers were located in different exons. Primer sequences were: GBP-1-FW: GGTCCAGTTGCTGAAAGAGC and GBP-1-RE: TGACAGGAAGGCTCTGGTCT. PCR conditions were: $95^{\circ} \mathrm{C}$ for $5 \mathrm{~min}$ followed by 37 cycles of $95^{\circ} \mathrm{C}$ for $15 \mathrm{~s}$, $55^{\circ} \mathrm{C}$ for $30 \mathrm{~s}$, and $72^{\circ} \mathrm{C}$ for $45 \mathrm{~s}$ followed by $72^{\circ} \mathrm{C}$ for $10 \mathrm{~min}$. PCR products were separated by agarose gel electrophoresis.

\section{Cell fractionation}

T84 cells treated with $100 \mathrm{U} / \mathrm{ml} \mathrm{IFN-} \gamma$ for $24 \mathrm{~h}$ were washed in phosphate-buffered saline and equilibrated for $5 \mathrm{~min}$ at room temperature in buffer $\mathrm{S}(0.25 \mathrm{M}$ sucrose, $1 \mathrm{mM}$ imidazole, and $5 \mathrm{mM} \mathrm{MgCl}_{2}$ ). Cells were harvested in buffer $S$ containing $1 \mathrm{mM}$ dithiothreitol and a protease inhibitor cocktail (Sigma), incubated on ice for $15 \mathrm{~min}$ and then homogenized using a douncer. Cells were centrifuged at $800 \mathrm{~g}$ at $4{ }^{\circ} \mathrm{C}$. The supernatant was collected and the pellet of nuclei was washed three times with buffer $\mathrm{N}$ (10 mM Tris, $10 \mathrm{mM} \mathrm{NaCl}, 3 \mathrm{mM} \mathrm{MgCl}_{2}$, and $0.05 \% \mathrm{NP}-40$, $\mathrm{pH}$ 7.4), vortexed for $30 \mathrm{~s}$ and centrifuged at $800 \mathrm{~g}$ for $5 \mathrm{~min}$. The washed nuclei were lysed with RIPA buffer $(20 \mathrm{mM}$ Tris, $150 \mathrm{mM} \mathrm{NaCl}, 2 \mathrm{mM}$ EDTA, $2 \mathrm{mM}$ ethylene glycol tetraacetic acid, $1 \% \mathrm{Na}^{+}$deoxycholate, $1 \%$ Triton $\mathrm{X}-100$, and $0.1 \%$ sodium dodecyl sulfate, $\mathrm{pH}$ 7.4) and sonicated. The supernatant containing membrane and cytosol fractions was centrifuged at $4{ }^{\circ} \mathrm{C}$ for $45 \mathrm{~min}$ at $100,000 \mathrm{~g}$. The supernatant (cytosolic fraction) was collected and the pellet (membrane fraction) was resuspended in a buffer containing $25 \mathrm{mM}$ Tris, $150 \mathrm{mM} \mathrm{NaCl}, 1 \% \mathrm{NP}-$ 40, $4 \mathrm{mM}$ EDTA, $25 \mathrm{mM}$ sodium fluoride, and $1 \mathrm{mM}$ sodium orthovanadate, $\mathrm{pH} 7.4$ and then sonicated. Fractions were analyzed by immunoblotting. Purity of the fractions was controlled by western blots of marker proteins for each fraction: mouse anti-human $\beta 1$-integrin as membrane marker (BD Biosciences), mouse anti-human GM130 (BD Biosciences) as Golgi marker and rabbit anti-promyelocytic leukemia protein as marker for the nuclear fraction (ABBiotec, San Diego, CA).

\section{Western blotting}

Equal amounts of protein were separated by polyacrylamide gel electrophoresis and transferred electrophoretically to polyvinyli- dene fluoride membranes. After incubation in phosphate-buffered saline containing $5 \%$ skim milk for $1 \mathrm{~h}$ to block unspecific binding, the membranes were probed with rat anti-human GBP1 antibody (clone 1B1; Calbiochem, La Jolla, CA) or mouse anti$\alpha$-tubulin (clone B-5-1-2; Sigma) as loading control for $1 \mathrm{~h}$ at $37^{\circ} \mathrm{C}$, washed three times in phosphate-buffered saline containing $0.5 \%$ skim milk and $0.05 \%$ Tween 20 and incubated with species-specific peroxidase-conjugated secondary antibodies (Jackson-Laboratories, Bar Harbor, ME) for $1 \mathrm{~h}$ at $37^{\circ} \mathrm{C}$. Signals were visualized using BM chemiluminescence substrate (Roche) and Hyblot ECL films (Denville, Metuchen, NJ).

\section{Immunofluorescence and confocal microscopy}

Human epithelial cells were grown on glass cover slips in 24well format to $70 \%$ confluency and then incubated for $24 \mathrm{~h}$ in the presence or absence of $100 \mathrm{U} / \mathrm{ml} \mathrm{IFN-} \gamma$ (in case of SK-CO15 cells $100 \mathrm{U} / \mathrm{ml} \mathrm{IFN}-\gamma+10 \mathrm{ng} / \mathrm{ml} \mathrm{TNF}-\alpha$ ). Cells were fixed for $20 \mathrm{~min}$ in ice-cold ethanol and blocked for $1 \mathrm{~h}$ in $1 \%$ BSA and $10 \%$ normal goat serum followed by incubation with antiGBP-1 and mouse anti-CAR (clone RmcB, kind gift from Dr J Bergelson, Division of Infectious Diseases, Children's Hospital of Philadelphia, PA) for $1 \mathrm{~h}$. Cells were washed and incubated for $1 \mathrm{~h}$ with species-specific fluorescently labeled Alexa-555 and Alexa-488 antibodies (Invitrogen). Cover slips were mounted in ProLong Gold medium (Invitrogen) and examined using an Axiovert LSM510 confocal laser microscope (Zeiss). For tissue staining, $8 \mu \mathrm{m}$ frozen tissue sections were mounted on glass coverslips. Discarded human colon tissue samples $(n=3$ for each condition: control, active UC, and active CD) were derived from anonymous surgical resection specimens or colonoscopy biopsies (active sites of UC and CD without extensive ulceration) and approved by the Emory University Institutional Review Board on human subjects. Sections were fixed in 100\% ethanol $\left(-20^{\circ} \mathrm{C}, 20 \mathrm{~min}\right)$ and air dried. Fluorescent labeling and analysis was performed as described above. In addition, nuclei were stained for 1 min using ToPro-3 dye (Invitrogen) after incubation with secondary antibodies. Fluorescence pixel intensities of GBP-1 labeling have been quantified by analysing 10 different fields of each condition using the LSM-FCS software (Zeiss).

\section{SiRNA transfection}

SK-CO15 cells were grown to $50 \%$ confluence and then treated with IFN- $\gamma$ and TNF- $\alpha$. Gene knockdown ${ }^{41}$ was performed as described earlier ${ }^{23}$, using two different siRNAs (Qiagen): GBP-1_3, AAGGCATGTACCATAAGCTAA and GBP-1_6, ATGGCATGTACCATAAGC TA. A validated siRNA against CyB obtained from Dharmacon (Chicago, IL) served as control. Transfections were performed using HiPerfect (Qiagen) in OptiMEM I medium (Invitrogen) according to the manufacturer's protocol.

\section{Transepithelial electrical resistance measurement}

SK-CO15 cells were plated on collagen-coated permeable polycarbonate transwell filters (Costar) with $0.4 \mu \mathrm{m}$ pore size and $0.33 \mathrm{~cm}^{2}$ surface and grown to $50 \%$ confluence. Cells 
were treated with IFN- $\gamma$ and TNF- $\alpha$ for $24 \mathrm{~h}$ before siRNA transfection as described above. TER of epithelial monolayers was measured every $24 \mathrm{~h}$ using an EVOMX electrovoltmeter with an STX2 electrode (World Precision Instruments, Sarasota, FL). Six independent experiments in six filters each were performed.

\section{Paracellular flux assay}

SK-CO15 cells were prepared on transwell filters as described above. When differences in TER were measured (usually 3-4 days after transfection), monolayers were washed three times with Hanks' balanced salt solution buffer before $10 \mu \mathrm{l}$ of the tracer solution $(10 \mu \mathrm{g} / \mu \mathrm{l}$ fluorescein isothiocyanate-dextran of $4 \mathrm{kDa}$; Sigma) were added to the apical side of monolayers. After $24 \mathrm{~h}$ of incubation at $37^{\circ} \mathrm{C}$, media from the bottom chamber were collected and the amount of diffused dextran was measured in a fluorometer (excitation wavelength $=492 \mathrm{~nm}$ and emission wavelength $=520 \mathrm{~nm}$ ).

\section{Detection of apoptosis}

SK-CO15 cells were transfected with siRNA to GBP-1 or CyB in transwell filters. Apoptosis was analyzed when differences in TER were observed. Cells were harvested in lysis buffer and apoptosis was detected by western blot using caspase-3, caspase9, and PARP antibodies (Cell Signaling Technology, Danvers, MA). TUNEL staining was performed using TUNEL Label and TUNEL enzyme (Roche), according to the manufacturer's instructions. The caspase inhibitor Z-VAD (Calbiochem) was applied at a final concentration of $10 \mu \mathrm{M}$ where indicated with medium change after transfection and renewed every $24 \mathrm{~h}$ until changes in TER were observed. Quantification of TUNELlabeled cells was performed using ImageJ.

\section{Statistics}

Data are presented as the mean with standard error and were compared by Student's $t$-test.

\section{ACKNOWLEDGMENTS}

We thank Dr Susan Voss for expert cell culture, Moshe Bachar for technical assistance, Dr Jan-Michael Klapproth (Department of Gastroenterology, Emory University Hospital, Atlanta, GA) for providing tissue material, and Dr Jeffrey Bergelson (Division of Infectious Diseases, Children's Hospital of Philadelphia, PA) for providing anti-CAR mAb RmcB. This work was supported by grants (RO1) DK72564, DK61379, and DK079392 from the National Institutes of Health to CAP and Schn 1155/1-1 from the German Research Foundation (DFG) to M.S.

\section{DISCLOSURE}

The authors declared no conflict of interest.

(C) 2009 Society for Mucosal Immunology

\section{REFERENCES}

1. Cheng, Y.S., Colonno, R.J. \& Yin, F.H. Interferon induction of fibroblast proteins with guanylate-binding activity. J. Biol. Chem. 258, 7746-7750 (1983).

2. Naschberger, E., Bauer, M. \& Sturzl, M. Human guanylate-binding protein-1 (hGBP-1) characterizes and establishes a nonangiogenic endothelial cell activation phenotype in inflammatory diseases. Adv. Enzyme Regul. 45, 215-227 (2005).
3. Olszewski, M.A., Gray, J. \& Vestal, D.J. In silico genomic analysis of the human and murine guanylate-binding protein (GBP) gene clusters. $J$. Interferon Cytokine Res. 26, 328-352 (2006).

4. Prakash, B., Praefcke, G.J., Renault, L., Wittinghofer, A. \& Herrmann, C. Structure of human guanylate-binding protein-1 representing a unique class of GTP-binding proteins. Nature 403, 567-571 (2000).

5. Guenzi, E. et al. The guanylate-binding protein-1 GTPase controls the invasive and angiogenic capability of endothelial cells through inhibition of MMP-1 expression. EMBO J. 22, 3772-3782 (2003).

6. Lubeseder-Martellato, C. et al. Guanylate-binding protein-1 expression is selectively induced by inflammatory cytokines and is an activation marker of endothelial cells during inflammatory diseases. Am. J. Pathol. 161, 1749-1759 (2002).

7. Guenzi, E. et al. The helical domain of GBP-1 mediates the inhibition of endothelial cell proliferation by inflammatory cytokines. EMBO J. 20, 5568-5577 (2001).

8. Anderson, S.L., Carton, J.M., Lou, J., Xing, L. \& Rubin, B.Y. Interferoninduced guanylate-binding protein-1 (GBP-1) mediates an antiviral effect against vesicular stomatitis virus and encephalomyocarditis virus. Virology 256, 8-14 (1999).

9. Itsui, Y. et al. Expressional screening of interferon-stimulated genes for antiviral activity against hepatitis $C$ virus replication. J. Viral. Hepat. 13, 690-700 (2006).

10. Pammer, J. et al. Interferon-alpha prevents apoptosis of endothelial cells after short-term exposure but induces replicative senescence after continuous stimulation. Lab. Invest. 86, 997-1007 (2006).

11. Nantais, D.E., Schwemmle, M., Stickney, J.T., Vestal, D.J. \& Buss, J.E. Prenylation of an interferon-gamma-induced GTP-binding protein: the human guanylate-binding protein, huGBP1. J. Leukoc. Biol. 60, 423-431 (1996).

12. Modiano, N., Lu, Y.E. \& Cresswell, P. Golgi targeting of human guanylatebinding protein-1 requires nucleotide binding, isoprenylation, and an IFNgamma-inducible cofactor. Proc. Natl. Acad. Sci. USA 102, 8680-8685 (2005).

13. Kumar, S. et al. Messenger ribonucleic acid encoding interferon inducible guanylate-binding protein-1 is induced in human endometrium within the putative window of implantation. J. Clin. Endocrinol. Metab. 86, 2420-2427 (2001).

14. Li, Q. et al. Interferon-gamma and tumor necrosis factor-alpha disrupt epithelial barrier function by altering lipid composition in membrane microdomains of tight junction. Clin. Immunol. 126, 67-80 (2008).

15. O'Connell, J. et al. Interferon-gamma sensitizes colonic epithelial cell lines to physiological and therapeutic inducers of colonocyte apoptosis. J. Cell. Physiol. 185, 331-338 (2000).

16. Yang, H., Fan, Y. \& Teitelbaum, D.H. Intraepithelial lymphocyte-derived interferon-gamma evokes enterocyte apoptosis with parenteral nutrition in mice. Am. J. Physiol. Gastrointest. Liver Physiol. 284, G629-G637 (2003).

17. Jarry, A. et al. Mucosal IL-10 and TGF-beta play crucial roles in preventing LPS-driven, IFN-gamma-mediated epithelial damage in human colon explants. J. Clin. Invest. 118, 1132-1142 (2008).

18. Bouma, G. \& Strober, W. The immunological and genetic basis of inflammatory bowel disease. Nat. Rev. Immunol. 3, 521-533 (2003).

19. Podolsky, D.K. Inflammatory bowel disease. N. Engl. J. Med. 347, 417-429 (2002).

20. Stoll, M. et al. Genetic variation in DLG5 is associated with inflammatory bowel disease. Nat. Genet. 36, 476-480 (2004).

21. Cho, J.H. \& Abraham, C. Inflammatory bowel disease genetics: Nod2. Annu. Rev. Med. 58, 401-416 (2007).

22. Goodlett, D.R. \& Aebersold, R. Correlation of mRNA and protein expression. In Analysing Gene Expression. A Handbook of Methods, Possibilities and Pitfalls (Lorkowski, S. \& Cullen, P., eds) 58-63 (WileyVCH, Weinheim, 2002).

23. Mandell, K.J., Babbin, B.A., Nusrat, A. \& Parkos, C.A. Junctional adhesion molecule 1 regulates epithelial cell morphology through effects on beta1 integrins and Rap1 activity. J. Biol. Chem. 280, 11665-11674 (2005).

24. Fish, S.M., Proujansky, R. \& Reenstra, W.W. Synergistic effects of interferon gamma and tumour necrosis factor alpha on T84 cell function. Gut 45, 191-198 (1999).

25. Coyne, C.B. \& Bergelson, J.M. CAR: a virus receptor within the tight junction. Adv. Drug. Deliv. Rev. 57, 869-882 (2005).

26. Vincent, T., Pettersson, R.F., Crystal, R.G. \& Leopold, P.L. Cytokinemediated downregulation of coxsackievirus-adenovirus receptor in endothelial cells. J. Virol. 78, 8047-8058 (2004). 


\section{ARTICLES}

27. Tewari, M. et al. Yama/CPP32 beta, a mammalian homolog of CED-3, is a CrmA inhibitable protease that cleaves the death substrate poly(ADPribose) polymerase. Cell 81, 801-809 (1995).

28. Naschberger, E. et al. Human guanylate-binding protein- 1 is a secreted GTPase present in increased concentrations in the cerebrospinal fluid of patients with bacterial meningitis. Am. J. Pathol. 169, 1088-1099 (2006).

29. Decker, T., Lew, D.J., Cheng, Y.S., Levy, D.E. \& Darnell, J.E. Jr Interactions of alpha- and gamma-interferon in the transcriptional regulation of the gene encoding a guanylate-binding protein. EMBO J. 8, 2009-2014 (1989).

30. Saunders, N.A., Smith, R.J. \& Jetten, A.M. Regulation of guanylatebinding protein expression in interferon-gamma-treated human epidermal keratinocytes and squamous cell carcinoma cells. J. Invest. Dermatol. 112, 977-983 (1999).

31. Furuta, G.T. et al. Hypoxia-inducible factor 1-dependent induction of intestinal trefoil factor protects barrier function during hypoxia. J. Exp. Med. 193, 1027-1034 (2001).

32. De Buhr, M.F. et al. Cd14, Gbp1, and Pla2g2a: three major candidate genes for experimental IBD identified by combining QTL and microarray analyses. Physiol. Genomics 25, 426-434 (2006).

33. Farmer, M.A. et al. A major quantitative trait locus on chromosome 3 controls colitis severity in IL-10-deficient mice. Proc. Natl. Acad. Sci. USA 98, 13820-13825 (2001).
34. Mahler, M. et al. Genetics of colitis susceptibility in IL-10-deficient mice: backcross vs. F2 results contrasted by principal component analysis. Genomics 80, 274-282 (2002).

35. Briken, V. et al. Interferon regulatory factor 1 is required for mouse Gbp gene activation by gammainterferon. Mol. Cell. Biol. 15, 975-982 (1995).

36. Staeheli, P., Prochazka, M., Steigmeier, P.A. \& Haller, O. Genetic control of interferon action: mouse strain distribution and inheritance of an induced protein with guanylate-binding property. Virology 137, 135-142 (1984).

37. Degrandi, D. et al. Extensive characterization of IFN-induced GTPases mGBP1 to mGBP10 involved in host defense. J. Immunol. 179, 77297740 (2007).

38. Lisanti, M.P., Caras, I.W., Davitz, M.A. \& Rodriguez-Boulan, E. A glycophospholipid membrane anchor acts as an apical targeting signal in polarized epithelial cells. J. Cell. Biol. 109, 2145-2156 (1989).

39. Parkos, C.A. et al. CD47 mediates post-adhesive events required for neutrophil migration across polarized intestinal epithelia. J. Cell. Biol 132, 437-450 (1996).

40. Schnoor, M. et al. Production of type VI collagen by human macrophages: a new dimension in macrophage functional heterogeneity. J. Immunol 180, 5707-5719 (2008).

41. Elbashir, S.M. et al. Duplexes of 21-nucleotide RNAs mediate RNA interference in cultured mammalian cells. Nature 411, 494-498 (2001). 